\title{
Isolated aldosterone deficiency in two infants: Mistakes and dilemmas in the diagnosis and treatment of a rare disease
}

\author{
Ivana Pela*, Laura Capirchio, Camilla Menchini, Giulia Anzilotti, Salvatore Seminara \\ Pediatric Endocrinology Unit, Department of Health Sciences, Meyer Children's Hospital, University of Florence, Florence, Italy \\ Email: ivana.pela@unifi.it
}

Received 7 November 2013; revised 28 November 2013; accepted 5 December 2013

Copyright (C) 2013 Ivana Pela et al. This is an open access article distributed under the Creative Commons Attribution License, which permits unrestricted use, distribution, and reproduction in any medium, provided the original work is properly cited.

\begin{abstract}
In this article, we describe the clinical picture and follow-up of two children diagnosed as suffering from pseudohypoaldosteronism when they were infants, and it was later recognized as isolated aldosterone deficiency in both. We illustrate the clinical differences between the two patients in terms of hydroelectrolytic balance, laboratory data and growth. In fact, while the growth and hematological parameters of the electrolytes and acid-base balance were normal in the first patient, and also without treatment with fludrocortisone thanks to very high renin activity, in the second patient, this treatment was vitally necessary to maintain normal growth and biochemical data. Despite the absence of a molecular analysis which could have confirmed this diagnosis, we believe that the description of the clinical evolution of these two cases from the moment of the incorrect diagnosis until the correct diagnosis and action taken, could be useful to highlight the extreme clinical variability of this rare disease.
\end{abstract}

Keywords: Hyponatremia; Salt-Wasting Syndrome; Metabolic Acidosis; Aldosterone; Renin

\section{INTRODUCTION}

Aldosterone is a key hormone which, through its effects on the Na-K ATPase pump of the distal convolute tubules, regulates electrolyte excretion and intravascular volume. Aldosterone expression is finely regulated through its classical pathway by the renin-angiotensin system. Renin is a proteolytic enzyme secreted by the juxtaglomerular apparatus of the nephron in response to various stimuli, such as $\beta$-adrenergic activity, low blood

"Corresponding author. pressure, sodium depletion, and above all, the decrease in intravascular volume, which cleaves angiotensinogen to angiotensin $\mathrm{I}$.

This is converted by the ACE (angiotensin-converting enzyme) into angiotensin II that binds to specific receptors of the cells of adrenal Zona glomerulosa, the site of aldosterone synthesis [1] (Figure 1).

Aldosterone synthesis deficiency may result from chronic primary adrenal insufficiency (Addison's disease) due to adrenal cortex injury after an infection, an autoimmune process, adrenal hemorrhage or infarction. However, it can also result from a genetic disorder involving the entire gland (such as congenital adrenal hypoplasia or X-linked adrenoleukodystrophy) or genetic disorders affecting converting enzymes that play a key role in aldosterone biosynthesis, such as the 21hydroxylase deficiency and the $3 \beta$-hydroxysteroid dehydrogenase deficiency. Generally these conditions are associated with combined deficits of gluco- and mineralcorticoid biosynthesis $[2,3]$.

Very few patients have aldosterone deficiency associated with entirely normal cortisol and sex steroid synthesis: indeed, this is a clinical condition called "isolated hypoaldosteronism", an inherited autosomal recessive disorder of terminal aldosterone synthesis, which leads to selective aldosterone deficiency. The prevalence of this rare disease is unknown, as well as its mortality, and

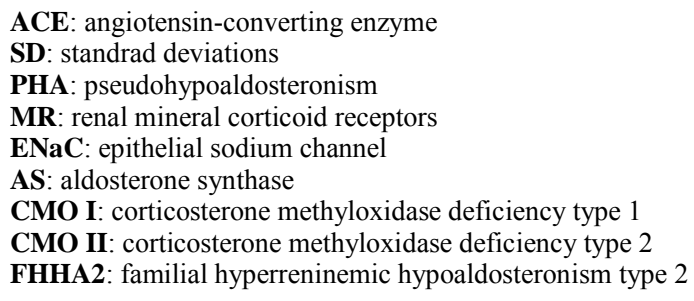

Figure 1. List of the abbreviations used in the text. 
it is possible that cases of sudden death could be attributed to acute electrolyte disorders consequent to the disease if not diagnosed and treated, especially in infancy. On the other hand, the prognosis of the patients correctly diagnosed and treated to prevent electrolyte disorders is generally good $[2,4]$. The clinical presentation of the isolated hypoaldosteronism varies with age. In newborns and infants it appears as a salt-wasting syndrome, characterized by severe hyponatremia, hyperkalemia, metabolic acidosis and prerenal azotemia associated with vomiting and signs of dehydration and hypovolemia such as generalized hypotonia, hypotension and tachycardia. Children are usually diagnosed because of failure to thrive, anorexia, mild dehydration and electrolyte abnormalities. Growth retardation may persist throughout childhood while electrolytes usually were normalized by 4 years of age with a high-sodium diet [5]. For this reason, it is widely believed that after childhood the disease does not require any treatment, probably as a result of multiple factors, including a diet rich in sodium and a greater ability of the adult kidney to reabsorb sodium. However, the study of Lovas and colleagues shows that, in adults affected by the disease, the absence of treatment was associated with a rise in serum potassium levels together with asymptomatic orthostatic hypotension, rendering them potentially vulnerable if exposed to other stress such as dehydration or limitation of dietary salt intake [6].

We present 2 observations of the neonatal-wasting syndrome, the clinical history and follow-up of which are compatible with a diagnosis of isolated hypoaldosteronism.

\section{CASE REPORTS}

Case 1. Q.M., Caucasian female, was born at 40 weeks by natural delivery after an uneventful pregnancy. Her birth weight was $3.400 \mathrm{Kg}$. She breastfed for 4 months, followed by weaning with formula milk. From the first week she had frequent vomiting episodes, manifesting 30 minutes after feeds, and failure to thrive, so she was referred to our pediatric ward for investigations at the age of 4.5 months. She was pale and hypohydrated with normal genitalia; her weight was $4.4 \mathrm{~kg}\left(<3^{\circ} \mathrm{C}\right)$, while her birth weight was $3.4 \mathrm{~kg}\left(50^{\circ} \mathrm{C}\right)$. Blood parameters revealed moderate renal insufficiency with urea $67 \mathrm{mg} / \mathrm{dl}$ and creatinine $2 \mathrm{mg} / \mathrm{dl}$, severe hyponatremia ( $\mathrm{Na} 120$ $\mathrm{mEq} / \mathrm{L}$ ) with hyperkaliemia (K $5.9 \mathrm{mEq} / \mathrm{L}$ ) and low chloride plasma concentration $(\mathrm{Cl} 93 \mathrm{mEq} / \mathrm{L})$, greatly increased renin activity $(>50 \mathrm{ng} / \mathrm{ml} / \mathrm{h} ;$ nv $0.2-2.8$ $\mathrm{ng} / \mathrm{ml} / \mathrm{h})$ with normal levels of aldosterone $(0.60 \mathrm{nmol} / \mathrm{L}$; nv $0.10-0.80 \mathrm{nmol} / \mathrm{L}$ ). Plasma $17-\mathrm{OH}$ progesterone and cortisol levels were normal and excluded a diagnosis of adrenogenital syndrome. Intravenous infusion of saline began with a weight gain, improvement of her general condition and regular feeding. Despite normal aldosterone levels in the presence of a high renin activity, pseudohypoaldosteronism was suspected and the therapy with oral $\mathrm{NaCl}$ supplement started. Two weeks after admission she was discharged with daily $\mathrm{NaCl} 60 \mathrm{mEq}$ therapy with normal plasma sodium and potassium levels, although at the limit of the normal range $(\mathrm{Na} 132 \mathrm{mEq} / \mathrm{L}$, $\mathrm{K} 4.9 \mathrm{mEq} / \mathrm{L}$ ).

The follow-up was carried out by pediatric endocrinologists. The clinical examination was negative. The serial check of the biochemical parameters showed normal serum electrolyte levels, so at the age of three years the $\mathrm{NaCl}$ supplements were discontinued. However, a persistent abnormally elevated renin activity $(>50$ $\mathrm{ng} / \mathrm{mL} / \mathrm{h}$ ) was observed, which was inappropriate for aldosterone levels in the presence of normal renal function, suggesting a diagnosis of primary isolated hypoaldosteronism. Therefore treatment with fludrocortisone was proposed. The parents refused because of her regular and normal growth, healthy condition and the normal biochemical parameters of acid-base and electrolyte balance; nevertheless, during follow-up, despite good hydration with normal plasma sodium, potassium and chloride levels, the renin activity remained persistently very high $(>50 \mathrm{ng} / \mathrm{ml} / \mathrm{h})$.

Case 2. F.A., Caucasian female, with intrauterine growth retardation was born to a toxic-dependant mother. Her birth weight was $2.250 \mathrm{~kg}$. A few hours after birth she suffered from tremors and irritability so was transferred to the Neonatal Intensive Care Unit and therapy with fenobarbital was introduced. During recovery she had frequent vomiting, dehydration with impaired renal function and failure to thrive. The blood examination revealed hyperkaliemia $(\mathrm{K} 6.26 \mathrm{mEq} / \mathrm{L})$, hyponatriemia $(\mathrm{Na} 127 \mathrm{mEq} / \mathrm{L})$, a slightly increased aldosterone level $(219 \mathrm{pg} / \mathrm{mlL}$; nv $10-160 \mathrm{pg} / \mathrm{mlL})$ with a greatly increased renin activity (74.2 and a maximum of $154.8 \mathrm{ng} / \mathrm{mL} / \mathrm{h} ; \mathrm{nv} 0.2-8 \mathrm{ng} / \mathrm{mL} / \mathrm{h}$ ), and with normal cortisol, 17-OH progesterone and ACTH levels. Transtubular potassium gradient was 1.1 showing low tubular efficiency in potassium excretion [7]. She was discharged four months later with the diagnosis of pseudohypoaldosteronism type 1 (PHA1) and therapy with $\mathrm{NaCl}$, bicarbonates and Kayexalate (ion-exchange resins).

During the follow-up, periodic blood examinations always revealed normal sodium and potassium concentrations with sporadic modest metabolic acidosis. An attempt at discontinuing therapy at the age of 30 months was unsuccessful because $12 \mathrm{~h}$ later she suffered from vomiting, weight loss, decreased sodium levels and increased potassium. The clinical examination was negative except for hyposomia; despite good metabolic equilibrium, her growth was seriously impaired and at 
the age of 36 months she was hospitalized in order to conduct further examinations. Her weight was $10.800 \mathrm{~kg}$ $(<-3 S D)$, and length $86 \mathrm{~cm}(<-3 S D)$, target height $154.5 \pm 6 \mathrm{~cm}$. The therapy with $\mathrm{NaCl}(2 \mathrm{mEq} / \mathrm{ml} 4$ times a day), Kayexalate $1.6 \mathrm{~g} 4$ times a day and $\mathrm{NaHCO}_{3}$ (3 $\mathrm{mEq} 4$ times per day) was discontinued and her biochemical parameters reassessed. Blood tests revealed hyponatremia ( $\mathrm{Na} 130 \mathrm{mmol} / \mathrm{L})$, normal kalemia (K 4.6 $\mathrm{mmol} / \mathrm{L})$, increased urea $(55 \mathrm{mg} / \mathrm{dl})$, metabolic acidosis (pH: 7.25; $\mathrm{pCO}_{2} 47.9 \mathrm{mmHg} ; \mathrm{HCO}_{3}^{-} 18 \mathrm{mmol} / \mathrm{L} ; \mathrm{ABE}$ - $6.7 \mathrm{mmol} / \mathrm{L})$; a normal-high aldosterone level $(0.44$ $\mathrm{nmol} / \mathrm{L}$, nv $0.020-0.44 \mathrm{nmol} / \mathrm{L})$ in the presence of a very increased renin activity (421 $\mathrm{microU} / \mathrm{ml}$; nv 2.8 $39.9 \mathrm{microU} / \mathrm{ml}$ ) with normal cortisol, 17-OH-progesterone and ACTH levels.

As a result of these data, the final diagnosis was changed to primary isolated ipoaldosteronism and treatment with fludrocortisone administered at a dosage of $0.05 \mathrm{mg}$ twice daily as soon as the previous symptomatic therapy was discontinued. During the follow-ups, her biochemical blood parameters moved steadily within the normal range and catch-up growth occurred, so at the age of 5, her weight was $16.000 \mathrm{~kg}(-1.88 S D)$ and her length $101 \mathrm{~cm}(-1.88 S D)$, according to target height.

\section{DISCUSSION}

The two infants were suffering from vomiting, failure to thrive, and dehydratation, all clinical features ascribable to salt-wasting syndromes. The blood parameters, similar in both patients, were characterized by hyponatremia, hyperkaliemia, normal chloride concentration, metabolic acidosis, and permanently very high renin activity in the presence of variable serum aldosterone levels, which were elevated during the episodes of severe dehydration as can be observed in Case 2, but within the normal range after recovering the normal hydro-electrolytic balance. However, the aldosterone serum levels were always "inappropriate" with respect to the strong renin stimulus. This clinical and metabolic picture amounts to a deficiency of aldosterone biosynthesis.

The most common inherited disorder of aldosterone synthesis that leads to the salt-wasting syndrome is the "classical form" of congenital adrenal hyperplasia (adrenogenital syndrome) due to 21-hydroxylase deficiency [8]. These patients cannot efficiently synthesize cortisol and consequently the adrenal cortex is overstimulated by ACTH, overproducing cortisol precursors. Some of these precursors are diverted to sex hormone synthesis, causing excessive secretion of adrenal androgens and consequent genitalia virilization in affected females. Infants with this salt-wasting syndrome may have signs and symptoms of adrenal insufficiency within the first 2 weeks of life, due to insufficient aldosterone synthesis [8]. However, the absence of external abnormal genitalia in both our cases and, overall, the normal 17-OH progesterone levels allowed for excluding a diagnosis of the adrenogenital syndrome.

Another possible cause of the salt-wasting syndrome is pseudohypoaldosteronism (PHA), a clinical condition in which failure to thrive, hyperkaliemia and metabolic acidosis, urinary sodium wasting, hyperreninemia and very elevated aldosterone levels are the leading clinical features. The most common form of pseudohypoaldosteronism is PHA type 1, which is characterized by normal adrenal responsiveness but apparent unresponsiveness of the renal tubules to the action of aldosterone. It consists of two forms: PHA type 1a and PHA type 1b. PHA type 1a, which is an autosomal dominant (AD) inherited disorder and only involves the renal mineral corticoid receptors (MR), manifests with primary renal tubular salt-wasting and is generally treated with sodium supplementation, sodium bicarbonate and, if necessary, potassium-binding resins. PHA type $1 \mathrm{~b}$ is an autosomal recessive (AR) inherited disorder and can be caused by homozygous or compound heterozygous mutation in any one of 3 genes encoding the subunits of the epithelial sodium channel $(\mathrm{ENaC})$; it is characterized by renal salt-wasting and high concentrations of sodium in sweat, stools, and saliva because aldosterone resistance is not restricted to the kidneys, but involves multiple organ systems and is particularly life-threatening during the neonatal period [9-11]. In case 2, the first diagnosis was PHA type 1a, based on a very high renin activity and, although to a lesser extent, aldosterone levels, therefore specific treatment was commenced with sodium bicarbonate, sodium chloride supplements and potassiumbinding resins. However, despite the good metabolic response with normalization of the acid-base and potassium balance, growth still remained very impaired and at the age of 36 months, her weight and height percentiles were less than $3 S D$. Furthermore, the attempt to discontinue the therapy at the age of 30 months, as is often possible with PHA type 1a, failed and a diagnostic reassessment at the age of 36 months showed biochemical parameters in line with a diagnosis of primary hypoaldosteronism.

Isolated primary hypoaldosteronism is characterized by the salt-wasting syndrome, normal cortisol and 17$\mathrm{OH}$-progesterone levels, high renin activity and low or normal aldosterone levels. The aldosterone deficiency with normal cortisol and sexual hormone production is a rare congenital disease due to the disruption of the final step of the aldosterone synthesis involving aldosterone synthase (AS), which is uniquely expressed in the Zona glomerulosa of the adrenal cortex, thus preventing production of aldosterone in the other adrenocortical zones (Figure 2). 


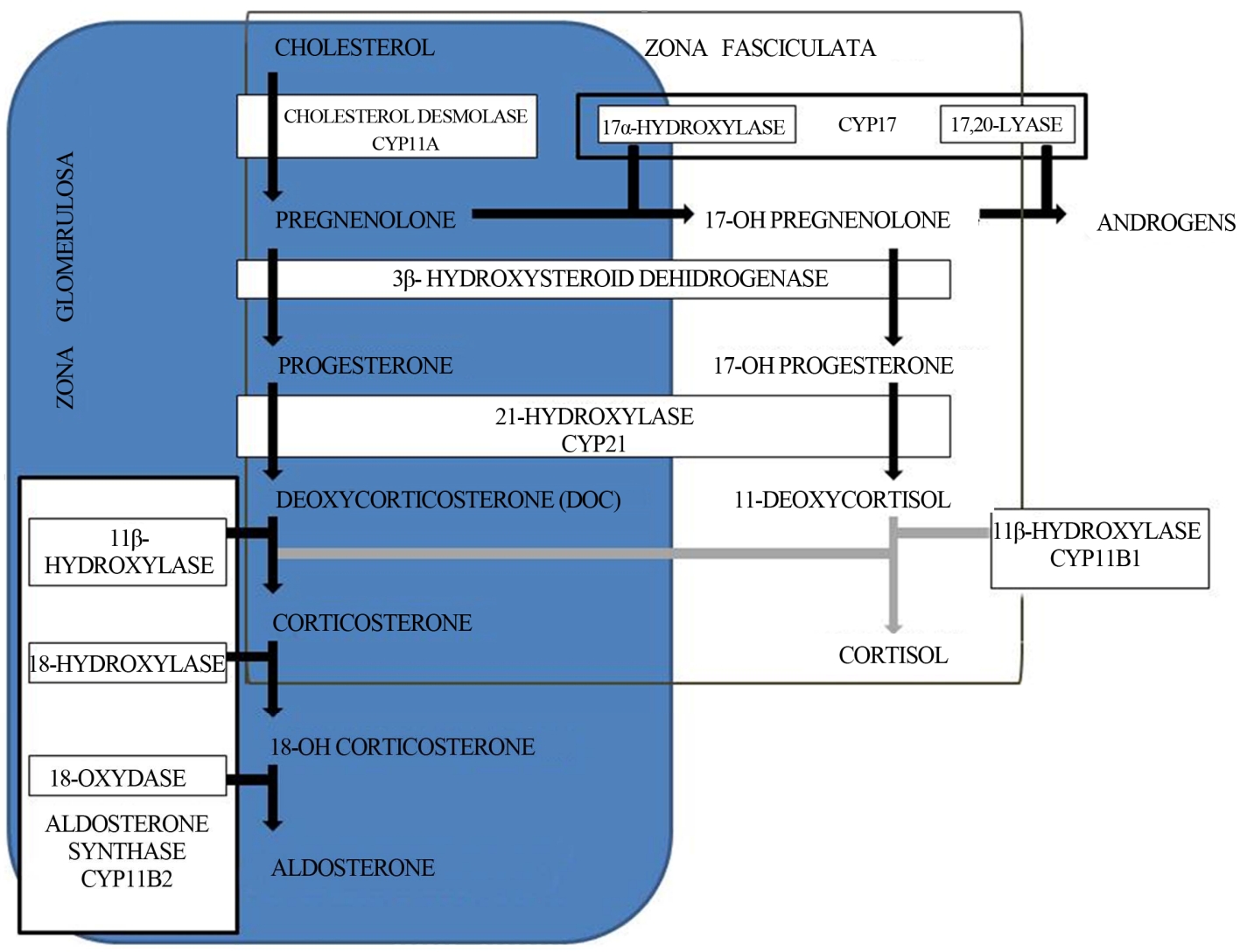

Figure 2. Biosynthetic pathway to aldosterone.

Two forms of aldosterone synthase deficiency have been recognized, also called corticosterone methyloxidase deficiency types 1 and 2 (CMO I and CMO II), with a similar clinical picture, but with different secreted steroids [1,5]. Both type 1 and type 2 of AS deficiency are autosomal recessive disorder caused by loss-offunction mutations in the CYP11B2 gene, a member of the cytochrome P450 family and localized in 8q24.3 $[12,13]$. The type of mutation in CYP11B2 and resulting residual enzymatic activity determines the phenotype, which is characterized by low to normal levels of 18-hydroxycorticosterone and undetectable aldosterone levels in type 1, and by high levels of 18-hydroxycorticosterone and subnormal or normal levels of aldosterone in type 2 [5]. It has been suggested that $11-\beta$ hydroxylase may be responsible for the production of 18-hydroxycorticosterone in type 2 AS deficiency, since this enzyme seems to have a weak 18-hydroxylation activity. In fact, the AS shows a $93 \%$ similarity with $11 \beta$-hydroxylase, expressed in the zona fasciculate and coded by the $C Y P 11 B 1$ gene, although weakly, the enzymatic activity of $C Y P 11 B 1$, and in some cases of $C Y P 11 B 2$ activity null could provide for the increased production of corticosterone (Figure 2) [4,5,14]. The two cases we describe here undoubtedly show the typical clinical and biochemical features of congenital primary hypoaldosteronism; therefore, because the aim of our study is to illustrate the likelihood of misunderstanding the biochemical parameters in case of severe hydroelectrolytic and metabolic disequilibrium due to this very rare disease, we can affirm his forcefulness. On the other hand, the study is not complete due to the unavailability of the dosage of 18-hydroxycorticosterone and corticosterone, which would have enabled better characterization of the defect, and above all, the molecular analysis which would have confirmed the diagnosis. However, it is known that no clear correlation has been demonstrated between the molecular defect and the clinical conesquences, but above all, that many cases with a clinical diagnosis of primary hypoaldosteronism show no mutations in the $C Y P 11 B 2$ gene, probably due to involvement of a trans-acting factor affecting CYP11B2 expression and/or development of the adrenal Zona glomerulosa [2]. This condition is known as familial hyperreninemic hypoaldosteronism type 2 (FHHA2).

We also wish to stress another aspect arising from our two cases. The widespread opinion regarding the evolution of primary hypoaldosteronism entails the possibility of suspending treatment in adolescent and adult patients because of the spontaneous improvement of the metabolic function of the renin-angiotensin system and the aldosterone activity on the renal tubulus [2]. 
Conversely, recent data from literature demonstrate that this condition needs lifelong treatment. In fact, the precarious metabolic stability gives rise to the risk of life-threatening metabolic disequilibrium in case of dehydration from various causes and when perfect performance of the renal system is required to control the hydroelectrolytic status [15]. Furthermore, despite the fact that discontinuation of treatment had no consequences on the electrolytes, acid-base or water balance, the very significant increase in the renin activity, as happened in Case 1, could cause renal disease, due to high angiotensin levels which, according to recent studies, produces glomerulosclerosis $[16,17]$.

Case 2 underscores another interesting aspect. It is known that in infancy and childhood, growth is strictly associated with good health, and also with a normal hydroelectrolytic balance. Treatment with sodium bicarbonate, sodium chloride and potassium-binding resins caused the normalization of the biochemical blood parameters, but growth was severely impaired and only the specific therapy with aldosterone analogue restored good physical development with catch-up growth. A similar experience is reported by Jessen and colleagues who note that fludrocortisone replacement therapy effectively normalizes sodium balance and growth in neonatal and infant age [18].

\section{CONCLUSION}

The cases we observed confirm the great variability of the clinical manifestations of isolated primary hypoaldosteronism, and emphasize the need for a reflection on the type and duration of treatment along with other data in literature. For this purpose, it is appropriate for patients with this rare disease to have a lifelong rigorous follow-up.

\section{REFERENCES}

[1] White, P.C. (1994) Disorders of aldosterone biosynthesis and action. New England Journal of Medicine, 331, 250257. http://dx.doi.org/10.1056/NEJM199407283310408

[2] Miller, W.L. (2005) The adrenal cortex and its disorders. In: Brook, C., Clayton, P. and Brown, R., Eds., Brook's Clinical Pediatric Endocrinology, 5th Edition, Blackwell Publishing, Malden, Massachussets, cp. 15, 293-351.

[3] Kayes-Wandover, K.M., Tannin, G.M., Shulman, D., Peled, D., Jones, K.L., Karaviti, L. and White, P.C. (2001) Congenital hyperreninemic hypoaldosteronism unlinked to the aldosterone synthase (CYP11B2) gene. The Journal of Clinical Endocrinology \& Metabolism, 86, 53795382. http://dx.doi.org/10.1210/jc.86.11.5379

[4] Ulick, S., Wang, J.Z. and Morton, D.H. (1992) The biochemical phenotypes of two inborn errors in the biosynthesis of aldosterone. The Journal of Clinical Endocrinology \& Metabolism, 74, 1415-1420. http://dx.doi.org/10.1210/jc.74.6.1415

[5] White, P.C. (2004) Aldosterone synthase deficiency and related disorders. Molecular and Cellular Endocrinology, 217, 81-87. http://dx.doi.org/10.1016/j.mce.2003.10.013

[6] Lovas, K., Mc Farlane, I., Nguyen, H.H., Curran, S., Schwabe, J., Halsall, D., Bernhardt, R., Wallace, A.M. and Chatterjee, V.K. (2009) A novel CYP11B2 gene mutation in an Asian family with aldosterone synthase deficiency. The Journal of Clinical Endocrinology \& Metabolism, 94, 914-919.

http://dx.doi.org/10.1210/jc.2008-1524

[7] Choi, M.J. and Ziyadeh, F.N. (2008) The utility of the transtubular potassium gradient in the evaluation of hyperkalemia. Journal of the American Society of Nephrology, 19, 424-426.

http://dx.doi.org/10.1681/ASN.2007091017

[8] White, P.C. and Bachega, T.A.S.S. (2012) Congenital adrenal hyperplasia due to 21 hydroxylase deficiency: From birth to adulthood. Seminars in Reproductive Medicine, 30, 400-409. http://dx.doi.org/10.1055/s-0032-1324724

[9] Saravanapandian, N., Paul, S. and Matthai, J. (2012) Pseudohypoaldosteronism type 1: A rare cause of severe dyselectrolytemia and cardiovascular collapse in neonates. Journal of Clinical Neonatology, 1, 224-226. http://dx.doi.org/10.4103/2249-4847.106007

[10] Throckmorton, D.G. and Bia, M.J. (1991) Pseudohypoaldosteronism: Report and discussion of the syndrome. The Yale Journal of Biology and Medicine, 64, 247-254.

[11] Riepe, F.G. (2013) Pseudohypoaldosteronism. In: Maghnie, M., Loche, S., Cappa, M. Ghizzoni, L. and Lorini, R., Eds., Hormone Resistance and Hypersensitivity. From Genetics to Clinical Management. Endocrine Development, Basel, Karger, 24, 86-95.

[12] Taymans, S.E., Pack, S., Pak, E., Torpy D.J., Zhuang, Z. and Stratakis, C.A. (1998) Human CYP11B2 (aldosterone synthase) maps to chromosome 8q24.3. The Journal of Clinical Endocrinology \& Metabolism, 83, 1033-1036. http://dx.doi.org/10.1210/jc.83.3.1033

[13] Bassett, M.H., White, P.C. and Rainey, W.E. (2004) The regulation of aldosterone synthase expression. Molecular and Cellular Endocrinology, 217, 67-74. http://dx.doi.org/10.1016/j.mce.2003.10.011

[14] Ulick, S. (1976) Diagnosis and nomenclature of the disorders of the terminal portion of the aldosterone biosynthetic pathway. The Journal of Clinical Endocrinology \& Metabolism, 43, 92-96.

http://dx.doi.org/10.1210/jcem-43-1-92

[15] Collinet, E., Pelissier, P., Richard, O., Gay, C., Pugeat, M., Morel, Y. and Stephan, J.L. (2012) Four cases of aldosterone synthase deficiency in childhood. Archives of Pediatrics, 19, 1191-1195. http://dx.doi.org/10.1016/j.arcped.2012.08.018

[16] Pearse, D.D., Tian, R., Nigro, J., Iorgulescu, J.B., Puzis, L. and Jaimes, E.A. (2008) Angiotensin II increases the expression of the transcription factor ETS-1 in mesangial cells. American Journal of Physiology. Renal Physiology, 294, 1094-1100. http://dx.doi.org/10.1152/ajprenal.00458.2007 
[17] Mezzano, S.A., Ruiz-Ortega, M. and Egido, J. (2001) Angiotensin II and renal fibrosis. Hypertension, 38, 635638. http://dx.doi.org/10.1161/hy09t1.094234

[18] Jessen, C.L., Christensen, J.H., Birkebaek, N.H. and Ritting, S. (2012) Homozygosity for a mutation in the
CYP11B2 gene in an infant with congenital corticosterone methyl oxidase deficiency type II. Acta Paediatrica, 101, 519-525.

http://dx.doi.org/10.1111/j.1651-2227.2012.02823.x 